\title{
Analyst
}

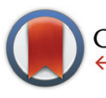

CrossMark

$\leftarrow$ click for updates

Cite this: Analyst, 2016, 141, 3590

Received 22nd January 2016,

Accepted 31st March 2016

DOI: 10.1039/c6an00152a

www.rsc.org/analyst

\section{Raman spectroscopy: an evolving technique for live cell studies}

\author{
Rachael Smith, ${ }^{a, b}$ Karen L. Wright ${ }^{\mathrm{b}}$ and Lorna Ashton*a
}

One of the most exciting developments in Raman spectroscopy in the last decade has been its application to cells and tissues for diagnostic and pharmaceutical applications, and in particular its use in the analysis of cellular dynamics. Raman spectroscopy is rapidly advancing as a cell imaging method that overcomes many of the limitations of current techniques and is earning its place as a routine tool in cell biology. In this review we focus on important developments in Raman spectroscopy that have evolved into the exciting technique of live-cell Raman microscopy and highlight some of the most recent and significant applications to cell biology.

\section{Introduction}

The cell is the basic biological unit of all living organisms, and the understanding of cellular dynamics and processes is vital for biological research as most diseases occur as a result of cellular abnormalities due to intracellular biochemical changes. ${ }^{1}$ Cell imaging allows these intracellular biochemical changes, along with normal behaviour, to be observed. Raman spectroscopy is an advancing cell imaging method that overcomes some of the limitations of current techniques.

Raman spectroscopy is a measure of the Raman effect, or inelastic scattering, first discovered by C. V. Raman in $1928 .^{2}$ He and K. S. Krishnan described the observation of a 'new type of secondary radiation' after experiments that involved illuminating samples with sunlight focused through a lens. They observed scattered light with a different wavelength to the original, incident wavelength; this scattering is now known as Raman scattering. This is induced by monochromatic light, usually from a laser, directed onto the cell; photons interact with this sample and energy can either be lost (Stokes) or gained (anti-Stokes). This difference in energy between the incident and scattered photon corresponds to the energy required to excite a particular molecular vibration; detection of these scattered photons produces a Raman spectrum, with different bands corresponding to the vibrational frequencies of different functional groups. Each molecule therefore has a unique fingerprint, or spectrum, according to the chemical bonds within it. $^{3}$

\footnotetext{
${ }^{a}$ Department of Chemistry, Lancaster University, LA1 4YG, UK.

E-mail: l.ashton@lancaster.ac.uk

${ }^{b}$ Faculty Health and Medicine, Division of Biomedical and Life Sciences, Lancaster University, Lancaster LA1 4YG, UK
}

While for cells these spectra can be complex, containing information about many different molecules and often requiring complex data interpretation, the potential of Raman spectroscopy in live cell imaging cannot be ignored. One of the key advantages of Raman spectroscopy is the ability to determine the underlying chemical structure of a cell: proteins, lipids and DNA can be visualised according to their vibrational spectra and as a result cells do not need to be labelled or stained prior to imaging. ${ }^{4}$ Furthermore, as water has a weak Raman signal, cells can be imaged within aqueous environments, meaning that live cell imaging is possible and making Raman spectroscopy an exciting alternative to existing imaging methods, allowing for the observation of living cells under normal physiological conditions.

Numerous other imaging techniques are currently used today in order to visualise cells. Historically, cell imaging was carried out only on fixed cells, primarily by electron microscopy. While providing valuable structural and biochemical information, these techniques only provide a 'snapshot' of the cells current state. ${ }^{5}$ In addition, cells often have to be stained prior to imaging, which has the potential to introduce artefacts and give an inaccurate representation of the natural intracellular state. The development of brightfield microscopy based techniques such as phase contrast and differential interference contrast microscopy meant that cells could be observed without staining, and live cell imaging became a possibility. ${ }^{6}$ Images obtained via these techniques can typically be collected more quickly than those acquired via Raman spectroscopy, but there is a lack of chemical specificity and there is the potential for artefacts to be introduced due to the halo affect. ${ }^{7}$

More recently, live cell imaging has been carried out using fluorescence microscopy, which allows for the detection of single molecules within a cell. Fluorescence microscopy is used to visualise the pattern of fluorescence within cells that 
have been stained with fluorescent molecules. The resolution of images achieved via fluorescence microscopy can be improved by techniques like STORM (stochastic optical reconstruction microscopy) or PALM (photo-activated localization microscopy) which sequentially selectively activate fluorophores throughout a single cell (for more detail on these techniques see ref. 8). Both of these techniques have been used in a number of live cell studies. ${ }^{9-11}$ FRET (fluorescence resonance energy transfer) is another fluorescence-related technique that can be used to detect and image protein-protein interactions within cells to give insight into cellular events like signal transduction and gene transcription. ${ }^{12}$ Whilst generally, these techniques have the advantage of giving a better resolution than can typically be achieved with Raman spectroscopy (down to 10 nm (ref. 13)), Raman spectroscopy has the advantage of increased chemical specificity and a lack of labels. Fluorescent microscopy relies on the expression of fluorescent molecules within cells, often by introducing external genes, ${ }^{6}$ which can directly affect cell physiology ${ }^{14,15}$ and as such, fluorescence imaging may not reflect the normal cellular environment in comparison to Raman-related techniques.

Infrared spectroscopy (IR) is another vibrational spectroscopy technique that can be utilised for live cell imaging. IR spectroscopy shares many of the advantages of Raman spectroscopy, in that it is non-invasive, non-destructive, and identifies the underlying chemical structure and as a result does not require labels. However, as water is a strong absorber of infrared radiation $^{3}$ this can make the analysis of substances in an aqueous environment difficult; Raman spectroscopy is therefore usually preferred over IR spectroscopy as cells can be maintained under physiological conditions. Additionally, Raman spectroscopy is typically able to achieve a better resolution. ${ }^{16}$

Due to its advantages over current techniques, there is a huge interest in developing Raman spectroscopy and imaging in biomedicine, with a particular emphasis on its potential for non-invasive in vivo disease diagnosis.

\section{Single cell Raman spectroscopy}

Currently, despite its advantages and potential, Raman spectroscopy is not widely used in clinical practice. This is primarily due to the fact that spontaneous Raman scattering is an inherently weak process, with only approximately $1 \times 10^{8}$ of photons inelastically scattered. ${ }^{3}$ Traditionally, this has resulted in long acquisition times and high laser intensity which is detrimental in examining biological samples and results in degradation. ${ }^{31}$ However, a number of new developments, techniques and instrumentation are starting to overcome these limitations (summarised in Fig. 1), leading to vast improvements and allowing for cell imaging via Raman spectroscopy to become a viable alternative to traditional methods. One of the most important step-changes in single cell Raman spectroscopy was the combining of a Raman spectrometer with a standard confocal microscope, the typical set-up of which can be seen in Fig. 2 below. In this technique, the laser beam is focused onto the cell by a microscope lens in order to give a much better resolution than can be achieved with traditional Raman spec- troscopy. ${ }^{26}$ By coupling the Raman spectrometer to a camera, usually a charged-coupled device (CCD), confocal Raman spectroscopy is able to image or map samples in addition to collecting their Raman spectra, allowing for the visualisation of intracellular components. ${ }^{32}$ Wide-field Raman imaging is a direct approach that illuminates the entire sample with a laser, and a narrowband filter is used to measure a specific wavenumber range. Raman mapping is more commonly used in cell studies, and traditionally involves raster-scanning a confocal laser spot and collecting the full Raman spectra sequentially at each location across the sample after dispersion onto the spectrometer CCD. ${ }^{23,33}$ A Raman map can them be computationally constructed to produce a pseudo-coloured image shaded according to the relative intensity of the Raman spectra at a given wavelength at each pixel. ${ }^{34}$ The first confocal Raman microscope was described in 1990, and was used to visualise and study single cells and chromosomes with a high spatial resolution. $^{26}$ Since 1990, many different studies have been carried out using confocal Raman spectroscopy to map cells.

As with many traditional cell imaging techniques, cells were initially fixed for these Raman spectroscopy experiments. Fixatives are used to preserve and to prevent the degradation of biological samples before they can be observed microscopically. The main fixatives used in Raman microscopy are alcohols (which fix samples by disrupting the hydrophobic bonds within proteins to denature them) and aldehydes (crosslinking fixatives that work by inducing covalent bonds between proteins). ${ }^{35}$ In fixed cells, Raman microspectroscopy has been used to map and visualise cell organelles, including: DNA by detecting the intensity of the $788 \mathrm{~cm}^{-1}$ band, associated with nucleic acids; ${ }^{36}$ proteins by measuring the bands for alkane groups at $2850 \mathrm{~cm}^{-1}, 2885 \mathrm{~cm}^{-1}$ and $2935 \mathrm{~cm}^{-1} ;^{37}$ lipid bodies by measuring the intensity of bands at $1658 \mathrm{~cm}^{-1}, 1267 \mathrm{~cm}^{-1}$ and $1411 \mathrm{~cm}^{-1} ;{ }^{38}$ and mitochondria by using bands at $750 \mathrm{~cm}^{-1}$, indicative of cytochrome $c$ distribution. ${ }^{39}$ In addition to this, studies on fixed cells have also been used to image cells at different stages of the cell cycle, ${ }^{40}$ to show subcellular changes that occur as a result of the stress-induced response, ${ }^{41}$ and to identify differences between benign and malignant breast tissue. ${ }^{42}$

However, whilst all of these above studies have provided valuable biochemical information, there is evidence that fixing procedures may alter the Raman spectra of cells in comparison to unfixed cells treated in the same way. Chan et al. reported changes in the intensity of bands associated with DNA, RNA, protein and lipid vibrations between fixed and unfixed cells, with methanol fixation showing greater changes than paraformaldehyde. ${ }^{43}$ A more recent study supported this, with ethanol inducing a greater change in the Raman spectra of nucleolar components than formaldehyde, ${ }^{44}$ and it has also been shown that formalin fixation induces changes in the Raman spectra of nucleic acids and proteins. ${ }^{45,46}$ Together, this data suggests that studies on fixed cells may not give accurate Raman spectra and has the potential to lead to data being misinterpreted, and studies on live cells are necessary to overcome this and to give more accurate results. 
C.V Raman's experiments on the colour of the sea, showing that the colour was due to molecular diffraction (1921)

C.V Raman and K.S Krishan discover the Raman effect (1928)

Monochromator invented

Monochromator invented
Observation of resonance Raman (RR)
spectra (1950)

First confocal scanning microscope built by Minsky (1955)

Lasers used as a light source for Raman spectrometers

Surface-enhanced Raman spectroscopy (SERS) observed by

Fleischman and colleagues (1974)

Gold and silver colloids used for SERS by Lee and Meisel (1982)

First confocal Raman spectrometer built (1990)

Single molecule SERS reported separately by Nie and Kneipp (1997)

3D CARS used for in in vivo tissue imaging (2005)

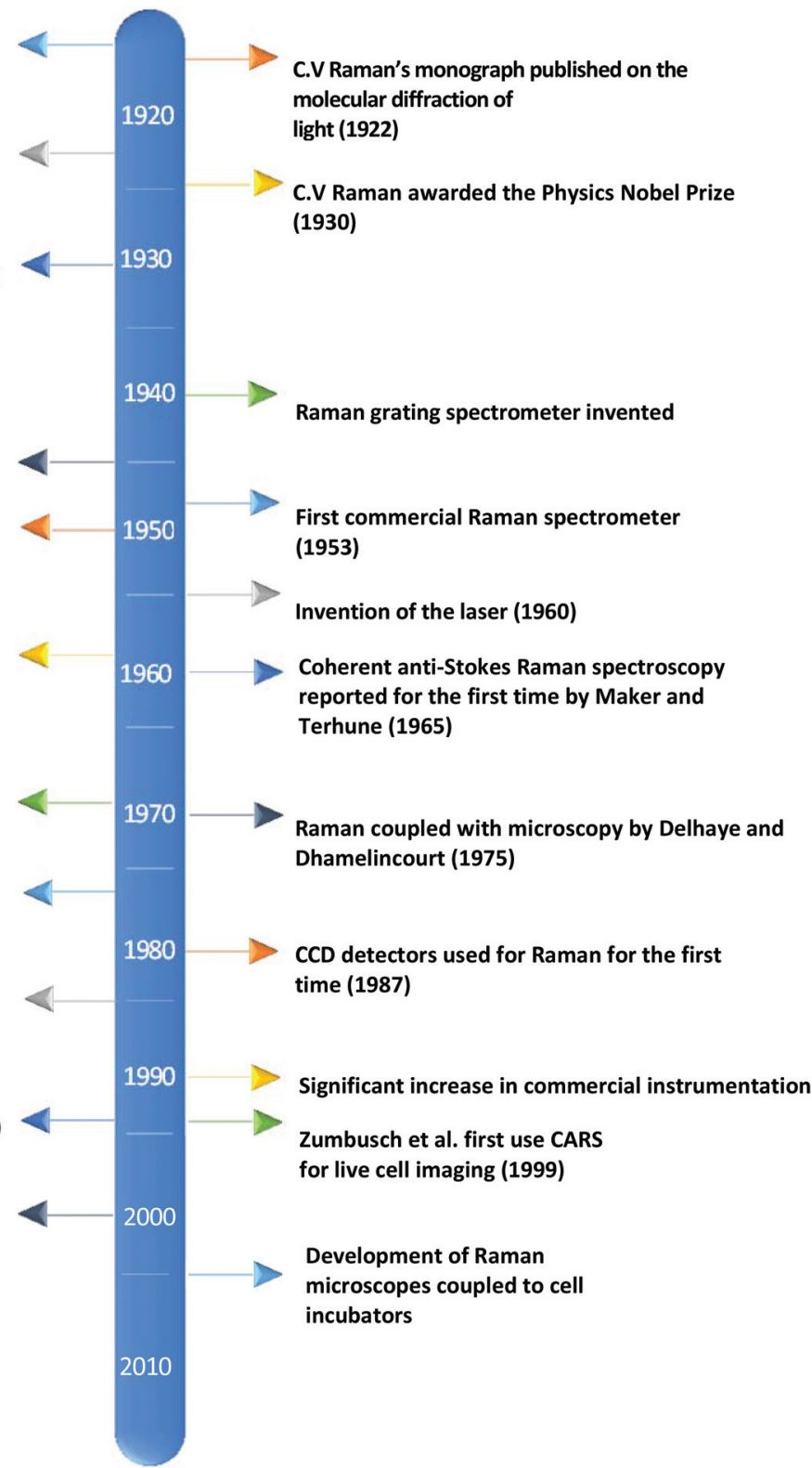

Fig. 1 A timeline of the key events in the history of Raman spectroscopy that have led to significant advancements in the field. 2,17-30

\section{Live-cell studies}

Raman microscopy is ideal for live cell studies, because it does not require the use of labels that may harm cells, and because it can be carried out in aqueous conditions due to the weak Raman intensity of water, cells can be imaged in either phosphate buffer solution (PBS) or medium, so do not need to be fixed prior to imaging. Many live cell studies use a laser with a wavelength of $785 \mathrm{~nm}$, just outside of the visible range. This is because there is evidence that visible wavelengths can cause degradation and photodamage to biological samples even at a low power and with short acquisition times. ${ }^{31,47}$ In contrast, it has been shown in several studies that a $785 \mathrm{~nm}$ laser does not cause this degradation, even with a high power and longer acquisition times, ${ }^{3,48}$ making it the preferred wavelength for the study of living cells.

Since the development of live-cell Raman imaging, many studies using living cells have been carried out and Raman microscopy has proved to be an important imaging technique in a number of different research areas. Initial Raman spectroscopy studies on live cells acquired spectra of cells immersed in PBS. One of the earliest studies occurred in 2005, when Wood et al. used Raman spectroscopy in order to image the oxygenation process of haemoglobin in human erythrocytes. Red blood cells were isolated from healthy volunteers and suspended in PBS before being transferred to a Petri dish for Raman measurements. Cells were imaged in the 1210-1250 $\mathrm{cm}^{-1}$ region over a period of 90 minutes; during 


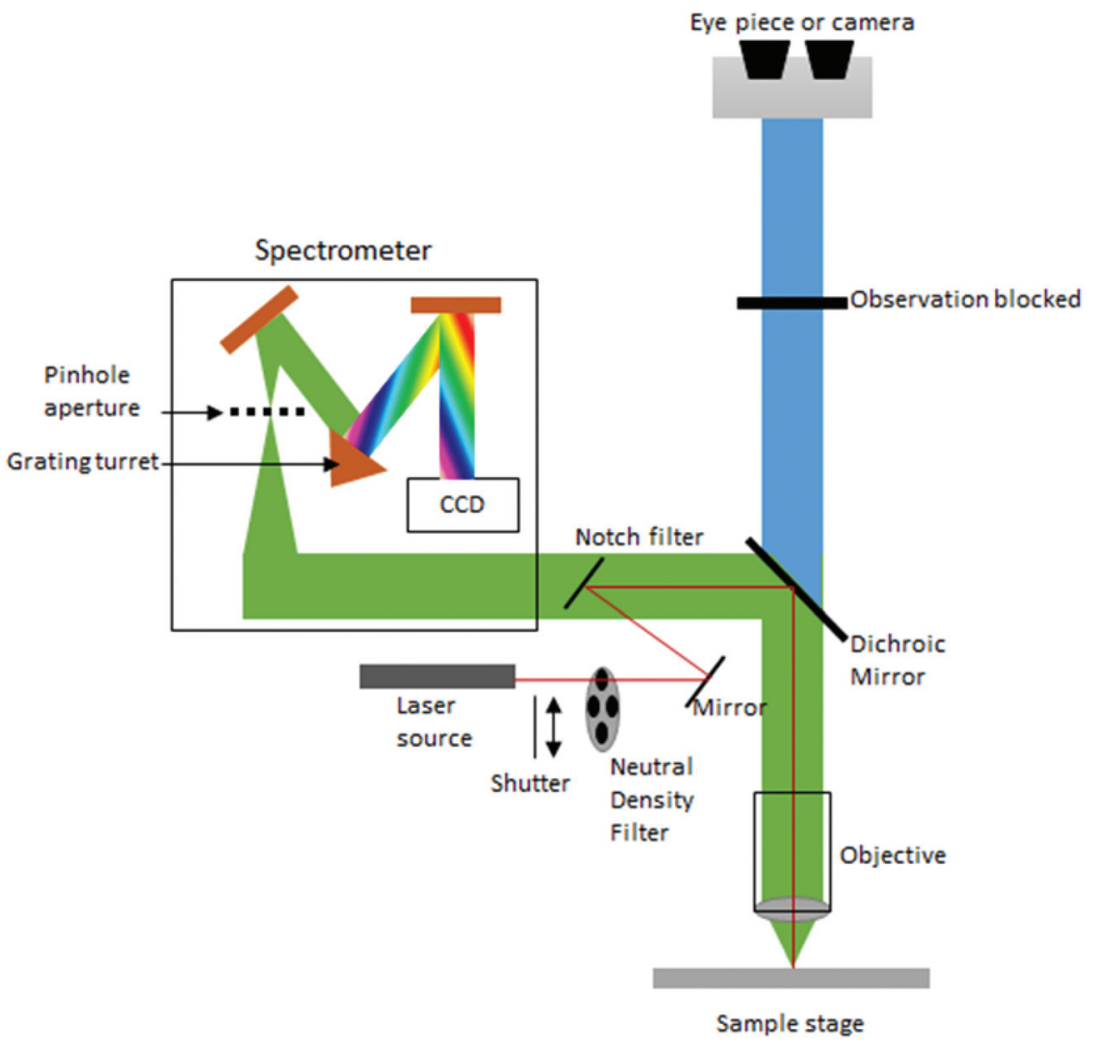

Fig. 2 Standard configuration of a confocal Raman microscope.

the first 50 minutes, cells were deoxygenated (by the addition of nitrogen), before being reoxygenated by atmospheric oxygen. Changes in the intensity of the measured band meant that the process could be followed and demonstrated the potential of Raman spectroscopy for single cell analysis of erythrocytes. ${ }^{47}$ Confocal Raman spectroscopy has also been applied to other cell types, and has been used to construct maps of lung carcinoma cells, ${ }^{49}$ and glioma cells, ${ }^{50}$ showing the distribution of nucleic acids, cell membrane lipids and proteins in both of these cell types. Both of these studies had an acquisition time of between 45-60 minutes, and while there was little loss of cell viability, ${ }^{49}$ this acquisition time is still long for biological samples and could be improved. More recent studies, including the investigation of hemozoin uptake in macrophages ${ }^{51}$ demonstrate that collection times can be reduced to 5-12 minutes depending on the cell size. The resonance effect can also be exploited in order to reduce acquisition times. In this technique, the frequency of the laser is adjusted so that it coincides with an electronic transition of the molecule of interest in order to enhance the Raman scatter and allow for high contrast spatial distribution imaging. ${ }^{3}$ This has been used in a number of Raman experiments to map the intracellular distribution of a number of different molecules in fixed cells, ${ }^{52,53}$ and more recently in living cells to visualise changes in cytochrome $c$ distribution during apoptosis. ${ }^{39}$ Studies such as these highlight the potential of Raman spectroscopy for not only determining different cell types, but also as an analytical tool to monitor biochemical changes in single cells in response to changes in the medium and cell culture conditions.

While studies in PBS have proved useful for studying biochemical processes, they do not give an accurate representation of normal physiological conditions, or as close as is possible in vitro. The need for in vitro models is great, especially in the field of drug development and cellular response to specific compounds, but also in terms of studying differences between normal and diseased or malignant cells and tissues in order to potentially develop Raman spectroscopy as a diagnostic tool. This has led to the development of Raman spectrometers that can be coupled to a cell incubator, allowing cells to be maintained under normal physiological conditions ( $37^{\circ} \mathrm{C}$ and $5 \%$ carbon dioxide). ${ }^{32}$ In 'true' live cell studies, cells are usually seeded directly onto the Raman substrate, held within sample holders or chambers - these are then immersed in medium and placed inside the incubator within the Raman microscope (Fig. 3). Several commercial microscope incubators are now available that include the incubator and the facilities to control the humidity, temperature and carbon dioxide and oxygen levels enabling cells to be kept alive for days at a time. Single cells have been mapped in this way by confocal microscopy to visualise the nucleus and cytoplasm of human breast cancer cells over a period of several hours, showing the potential of Raman spectroscopy in timecourse studies. ${ }^{32}$ 


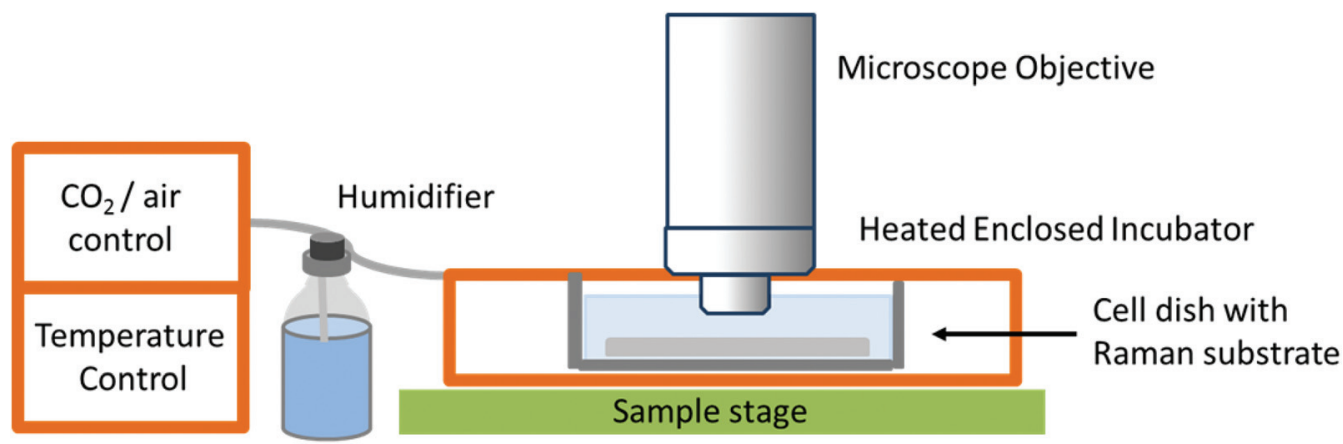

Fig. 3 Schematic of a typical live cell incubator set-up for combining with Raman spectroscopy. While this is the typical set up by commercial manufacturers, some live cell experiments for long-term studies use an inverted microscope as unlike this set-up, the microscope objective does not need to be immersed in media. ${ }^{32}$

At present there are two major limitations in the translation of live cell Raman spectroscopy from the laboratory into the clinic: difficulties in analysing the data and as previously mentioned slow acquisition times. There is often a misconception outside of the field of Raman spectroscopy that data analysis is difficult and time consuming for non-specialists. Although there is no standardized approach for how to do this, with different groups using different methods, ${ }^{34}$ rapid improvements in data analysis software supplied with Raman spectrometers is making it easier and quicker to convert the Raman maps into meaningful pictures. However, there is still a need for those carrying out single cell Raman spectroscopy to work together to establish robust and reliable protocols that are user-friendly and applicable to cell biology. As previously discussed, progress is being made with the second limitation of slow acquisition times.

One method to increase imaging speed as well as gaining increased chemical specificity is stable-isotope labelling (SILAC), primarily using amino acid isotopes. These can be easily incorporated into all newly synthesised proteins within cells by being added to the culture medium, and are an exciting labelling method as, unlike the fluorophores mentioned earlier in this review, there is little chemical difference between these isotopes and the natural amino acid, meaning that cellular behaviour will not be altered. ${ }^{54}$ The incorporation of stable isotopes within cells will shift the Raman band at a particular frequency which can be observed and used for labelling purposes. This technique has been used in a number of Raman-based studies to further investigate cell physiology and behaviour beyond the limitations of traditional Raman microscopic techniques. SILAC has been combined with Raman spectroscopy to show the previously unobserved localisation of newly synthesised proteins to lipid droplets in live yeast cells, ${ }^{55}$ to visualise the uptake and distribution of labelled lipids and the subsequent formation of cytosolic lipid droplets during the process of macrophage foam cell formation, ${ }^{56}$ to study carbon flow from an amino acid source to predatory nematodes via $E$. coli, ${ }^{57}$ and to, for the first time, observe the exchange of amino acids between a host cell and an infecting parasite, $T$. gondii, in real time. ${ }^{58}$
As well as improvements in collection times for conventional Raman spectroscopy two further Raman approaches have been shown to significantly reduce acquisition times: coherent anti-Stokes Raman spectroscopy (CARS) and surface enhanced Raman spectroscopy (SERS).

CARS is a nonlinear technique that measures the antiStokes shift rather than the Stokes shift. CARS achieves this by using a pump beam at frequency $\omega_{\mathrm{p}}$ and a Stokes beam at frequency $\omega_{\mathrm{s}}$ to tightly focus onto a cell, generating an antiStokes signal at a frequency of $2 \omega_{\mathrm{p}}-\omega_{\mathrm{s}}$. If this frequency is equal to the frequency of the molecular vibration of a particular chemical bond, then a strong signal is generated as all these molecules are converted into a vibrational state at the same time. ${ }^{59}$ As only a single vibrational frequency is examined at any one time, CARS is a highly selective imaging technique with a signal that is 5-6 orders of magnitude greater than that observed in the spontaneous Raman scattering process, meaning that CARS is particularly useful for the study of specific molecules within a cell. As traditional CARS is limited to study only a single Raman peak, it has most commonly been used to examine lipid distribution in cells, due to the abundance of $\mathrm{C}-\mathrm{H}$ bonds within lipids. It has been used to map lipid droplet distribution by measuring the vibrational frequency at $2845 \mathrm{~cm}^{-1}$, and to follow cellular differentiation of live fibroblast cells into fat cells over a period of several days. ${ }^{60}$ It has also been used to visualise the nuclear membrane during interphase and chromosomes during metaphase in living cells, as well as to observe the process of apoptosis, again in fibroblast cells. ${ }^{61}$

SERS is a technique that aims to increase the weak Raman intensity associated with spontaneous scattering, and in some cases has increased the intensity to such an extent that single molecule detection has been achievable. ${ }^{27}$ In SERS, a metallic nanostructure is used as a substrate (typically silver or gold); when this surface interacts with incident photons, an enhanced electric field is generated, increasing Raman scattering from molecules near to or adsorbed onto the substrate (reviewed in detail in ref. 62). For cell-based studies, cells can be incubated with a metal colloid suspension in order to give high resolution intracellular images with a short acquisition 
time. ${ }^{63}$ These nanoparticles can be targeted towards organelles of interest in order to map organelles within living cells, and this technique has been used in a number of different studies. Gold nanoparticles have been used in order to visualise the nucleus of human oral squamous carcinoma cells as they passed through the cell cycle over a period of 24 hours, showing clearly the changes in nuclear structure as cells progressed from $\mathrm{G} 1$ through to $\mathrm{M}$ phase. ${ }^{64}$ Another recent study used dye-coded gold nanoparticles modified with peptides to target the cytoplasm, mitochondria and nucleus of human oral cancer cells. Cells were incubated with these particles for either three, six or twelve hours prior to Raman spectral acquisition. The use of gold nanoparticles increased the Raman intensity by such a factor that acquisition times of $10 \mathrm{~ms}$ per pixel could be used to map mitochondria and the nucleus, resulting in a total spectral acquisition time of just 27.5 seconds. $^{65}$

It is worth noting that a number of other instrumental or sampling approaches can be used in order to increase imaging speed without resorting to CARS and SERS in order to obtain a full spectrum across the whole cell without the introduction of nanoparticles. Selective sampling can be used in order to reduce acquisition times, and involves taking the Raman spectra at selected points rather than raster scanning the whole sample, and is particularly useful in studies were only a particular part or structure of the cell is of interest. This methodology has been used in order to measure Raman spectra of a parasite, Neospora caninum, invading host cells, reducing acquisition times by up to ten times in comparison to raster scanning. ${ }^{66}$ Multi-focal scanning is another technique that can increase the speed of spectral acquisition, and has been able to acquire Raman maps of bacterial spores in $\sim 1$ minute. ${ }^{67}$

Together, these studies demonstrate the ability of Raman spectroscopy to provide valuable biochemical information from living cells in a relatively short space of time, allowing for the real-time observation and monitoring of cellular processes in vitro in a way that other current imaging techniques cannot.

\section{Current and potential applications of live-cell imaging}

Stem cell identification and characterisation. Stem cells are undifferentiated cells able to differentiate into a number of different cell lineages, and are important in a number of different research areas. With development, stem cell based therapies have the potential to be used to treat a number of different diseases, including (but not limited to): diabetes, liver disease, degenerative neural disease and muscular dystrophies. ${ }^{68}$ A major limitation within this field is the heterogeneous nature of stem cell cultures, as specific cell phenotypes are required for the treatment of different diseases, and the proliferation of undesirable phenotypes can be detrimental. ${ }^{69}$ Current techniques to identify and characterise stem cell phenotype are limited and need to be improved before stem cell isolation can be translated into the clinic. As Raman spectroscopy is a non-invasive and label-free technique it is already advantageous over current methods and allows for the characterisation of living cells in vitro.

Different cell types express different molecular markers, and these can be exploited in order to characterise and sort specific cell types from a heterogeneous population of cells. Pascut et al. utilised molecular markers specific for hESCderived cardiomyocytes, glycogen (band at $860 \mathrm{~cm}^{-1}$ ) and myofibril proteins (band at $938 \mathrm{~cm}^{-1}$ ), in order to specifically image cardiomyocytes in a population of other hESC-derived cells with a high level of accuracy for phenotypic identification. $^{70}$ This study demonstrated the potential of Raman spectroscopy in characterising hESC phenotype in a non-invasive manner, which is vital for the cell sorting and purification required for the clinical application of stem cell based therapies, but acquisition times were long and therefore inappropriate for translation into the clinic. However, the same group managed to decrease acquisition times to just 5 seconds per cell in a more recent study, ${ }^{71}$ making strides towards Raman spectroscopy mediated cell identification and sorting to be used in the clinic. The same technique has been applied to neural stem cells, using RNA content as a molecular marker (peak at $813 \mathrm{~cm}^{-1}$ ) in order to map them and to distinguish these cells from glial cells ${ }^{72}$ (see Fig. 4), demonstrating the versatility of this technique to detect different stem cell phenotypes.

More recently, Raman spectroscopy has been used to characterise the differentiation of adipose derived stem cells (ADSCs) over a period of 14 days $^{73}$ and dental pulp stromal cells (DPSCs) over a period of 28 days $^{74}$ under aseptic conditions. The authors used customised, sterilised cell culture flasks with quartz windows in order to achieve this, and showed that it is possible to follow the differentiation of these cells by monitoring key biochemical changes that occur during the process. The fact that the sterility of the culture was maintained in these experiments is significant, as the same cells can be expanded and used in further experiments, saving time and money over traditional characterisation techniques such as flow cytometry and immunocytochemistry. ${ }^{75}$ While these methods are not yet applicable to the clinic with a reduction in differentiation seen in one study ${ }^{73}$ and long acquisition times in the other, ${ }^{74}$ they further demonstrate the potential of Raman spectroscopy in the identification and characterisation of stem cell phenotype and, with further developments, may be able to be translated into the clinic.

\section{Pharmaceutical applications}

Perhaps one of the most exciting potential applications of Raman spectroscopy is in the study of pharmaceutical compounds. In vitro studies of drug distribution and cellular response can be monitored in real-time, potentially reducing both the financial burden of the early stages of drug development and the need for animal studies, especially in regards to toxicity testing. Apoptosis, the cellular response to toxic agents, can be detected by changes in the Raman spectra of cells, and visualised by Raman mapping. This technique has been used to show a build-up of lipids in the cytoplasm of eto- 


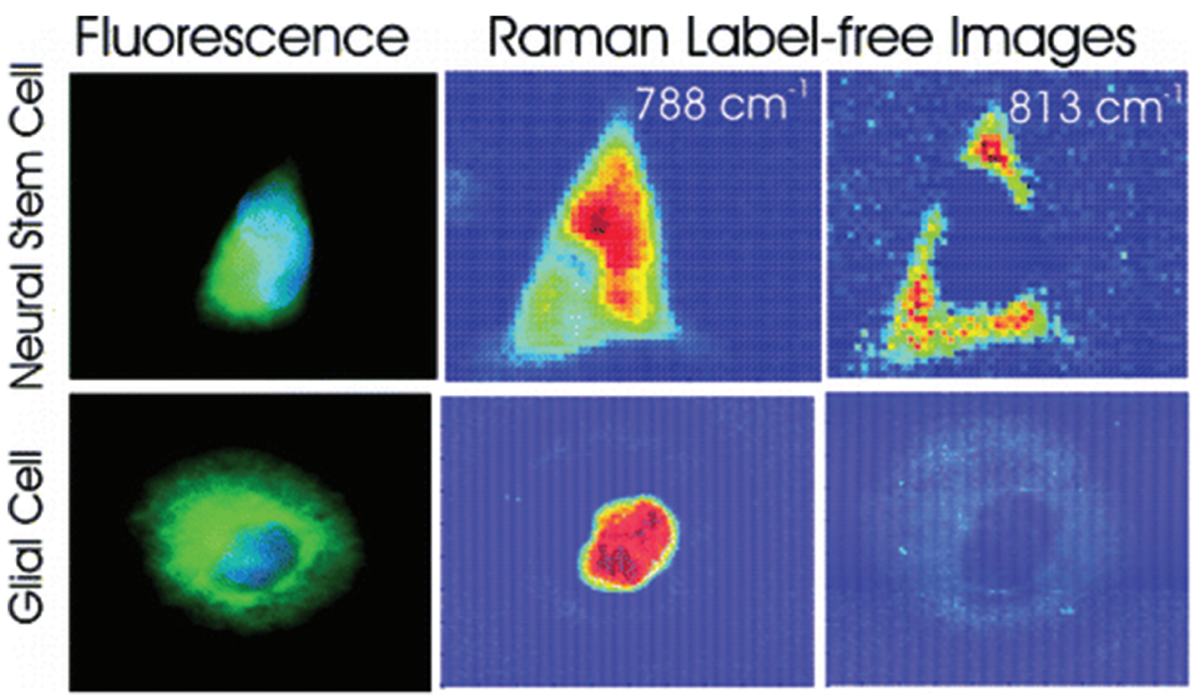

Fig. 4 Fluorescent and Raman images demonstrating the differences between glial and neural stem cells. The Raman band at $788 \mathrm{~cm}^{-1}$ is associated with the nucleic acids of DNA and RNA, whilst the band at $813 \mathrm{~cm}^{-1}$ is associated with RNA only. High levels of RNA are observed in the cytoplasm neural stem cells, but RNA is absent in the cytoplasm of glial cells, demonstrating that Raman spectroscopy can be used to non-invasively distinguish cell phenotype. Reproduced with permission from the American Chemical Society, Copyright 2012, ref. 72.

poside treated breast cancer cells, ${ }^{76}$ and the loss of cell volume and cell shrinkage associated with docetaxel treatment in a human adenocarcinoma cell line. ${ }^{77}$ If the drug of interest has a peak in its Raman spectra that differs from or is outside the range of the typical cellular components, then the drug itself can be visualised intracellularly in a label-free manner. This technique has been used to show the distribution of the anticancer drug, paclitaxel, in a breast cancer cell line by measuring the Raman peak at $1740 \mathrm{~cm}^{-1}$, representative of the $\mathrm{C}=\mathrm{O}$ bond vibration, to show the movement of the drug through the cell membrane and into the cytoplasm. ${ }^{78}$

SERS is a particularly useful technique in this field, as nanoparticles can be used not only to enhance the Raman signal, but to aid cellular uptake of the drug of interest and allow for targeted drug delivery. Gold-coated silver nanoparticles have been used to track the movement and cellular metabolism of an anti-tumour drug, 6MP, in a human lung adenocarcinoma cell line over a period of 24 hours, ${ }^{79}$ suggesting that SERS is an efficient method for studying drug efficacy (demonstrated in Fig. 5). In addition, SERS nanoparticles can be designed not only to be conjugated to the drug of interest, but also to have an outer layer that is $\mathrm{pH}$ sensitive, allowing for $\mathrm{pH}$-dependent drug release. This technique has been used to deliver doxorubin to living cervical cancer cells in vitro. The nanoparticles were conjugated with transferrin, which is overexpressed in these cells, for specific targeting, and SERS maps were constructed to show drug internalisation. ${ }^{80}$ As most cancer cells exist within an acidic environment due to the nature of their metabolism, ${ }^{81} \mathrm{pH}$-sensitive nanoparticles conjugated to toxic agents may represent a novel way to develop new anti-cancer treatments.

Together, all of the above studies show the potential of Raman microscopy in the development of pharmaceutical compounds, not only for toxicity studies but to assess intracellular distribution and the effect different drugs have on different cell lines in addition to allowing for targeted delivery, and suggest that Raman microscopy may be a feasible method of predicting the in vivo response of a drug in vitro.

\section{Diagnostics}

Another major potential application of Raman spectroscopy is in the diagnosis of disease. Many diseases originate from underlying biochemical changes within cells, and as Raman spectroscopy is able to detect these subtle changes, sometimes with a high level of sensitivity, its potential in diagnostics cannot be ignored. Raman spectroscopy could potentially be used in the diagnosis of a number of different diseases from atherosclerosis $^{82,83}$ to osteoarthritis, ${ }^{84}$ but it is particularly relevant in cancer research. As with fixed cells, Raman spectroscopy has been used to examine the differences between normal and neoplastic living cells, mainly by their Raman spectra, ${ }^{85,86}$ and SERS can be used to probe living cells for specific markers. Many cancer cells express proteins that are not usually found in normal cells of the same phenotype, and detection of these proteins may aid in the diagnosis of disease. The feasibility of SERS in this field has previously been demonstrated in several studies. Both silver ${ }^{87}$ and gold ${ }^{88}$ nanoparticles conjugated to an antibody specific for HER2, a receptor overexpressed on the surface of many breast cancer cells, have been used in order to map these cells live (see Fig. 6). There is the potential for this technique to be developed further and to be used in in vivo studies. Already, several studies have been conducted on murine models, primarily collecting Raman spectra rather than mapping individual cells themselves, ${ }^{89,90}$ and there is the real possibility that these techniques may be able to be developed further until they are clini- 
A
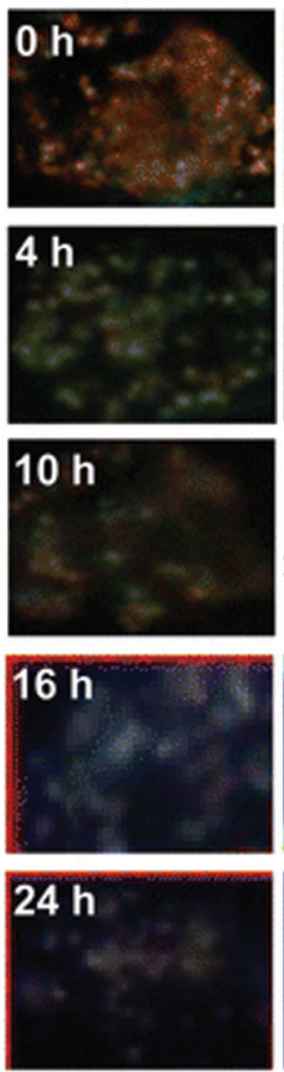

weak
B
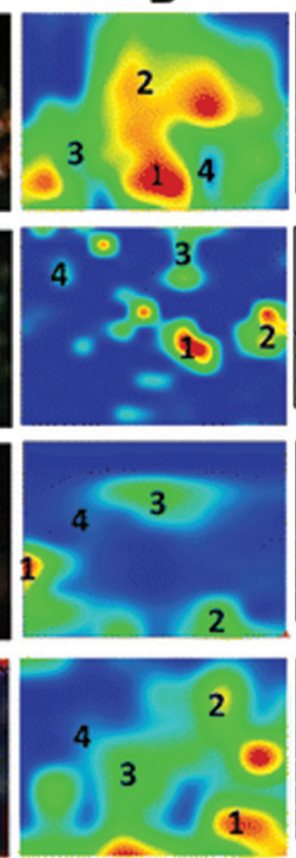

strong

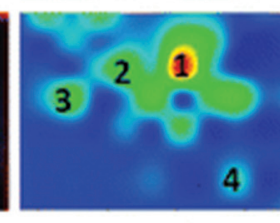

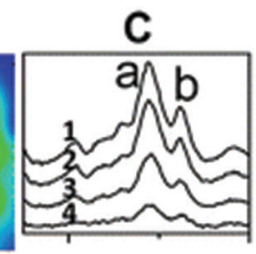
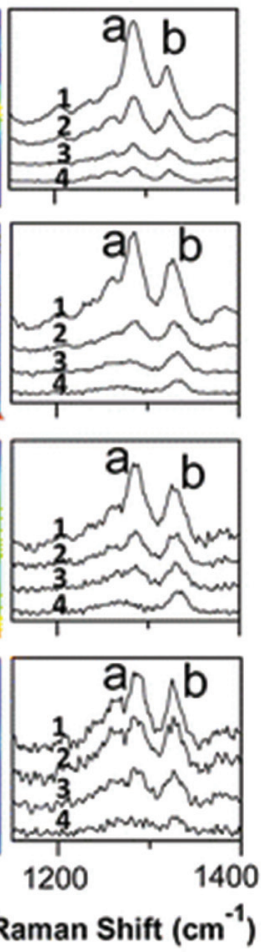

Fig. 5 Darkfield microscopy images (A), Raman maps (B) and Raman spectra (C) to show the metabolism of 6MP in A549 cells over a period of 24 hours. At 0 hour Raman intensity was mapped at $1286 \mathrm{~cm}^{-1}$ (peak a) to show the distribution of $6 \mathrm{MP}$. Subsequent maps use the Raman intensity of $1330 \mathrm{~cm}^{-1}$ (peak b), representative of 6MPR, one of the active anti-cancer agents formed from the breakdown of 6MP. 6MPR began to form at 4 hours, and the concentration increased with increasing time as the concentration of 6MP decreased. This demonstrates the successful development of a label-free Raman based methodology to monitor and visualise the metabolism of 6MP in living cells. Reproduced with permission from the American Chemical Society, Copyright 2014, ref. 79. (a)

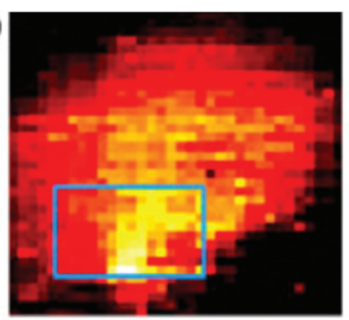

(b)

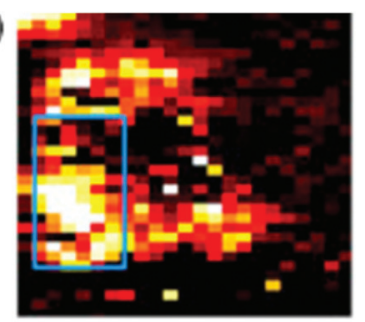

Fig. 6 SERS mapping images of a MCF7 cell expressing HER-2 using hollow gold nanoparticles (a) and silver nanoparticles (b) specific for HER-2 at $1620 \mathrm{~cm}^{-1}$, demonstrating the feasibility of using SERS probes for cancer detection. Reproduced, with permission from Elsevier, Copyright 2009, ref. 87. cally relevant. The combination of Raman spectroscopy and endoscopy has already been employed in the diagnosis of oesophageal cancer with an accuracy of $96 \%,{ }^{91}$ suggesting that, with further development in order to distinguish between healthy and cancerous tissue of other cells and tissues within the body, Raman spectroscopy could become an important clinical diagnostic tool in the future.

The use of SERS probes may also be particularly useful in the detection of cancerous cells within solution, such as blood or urine for non-invasive diagnosis of disease, ${ }^{92}$ with relevance to leukaemia, ${ }^{93}$ and bladder cancer. ${ }^{94}$ The development of optofluidic cell sorting systems has meant that single cells in solution can be identified and sorted according to their Raman spectra ${ }^{95}$ with a high specificity of $99 \%$ for the discrimination of leukocytes, leukemic cells, and two different breast cancer cell lines, ${ }^{96}$ giving further evidence towards the use of Raman spectroscopy in this type of diagnosis.

Overall, these studies demonstrate the potential of Raman spectroscopy in cancer diagnosis. Current diagnostic techniques do not give accurate biochemical information about a sample in the way that Raman spectroscopy is able to, and often rely on examining visual differences that may be misinterpreted. There is therefore an interest in developing more accurate techniques, and there is evidence that Raman spectroscopy may be a viable, label-free and non-invasive alternative.

\section{D imaging}

3D cell cultures are becoming an increasingly used and important in vitro model in a number of different biological applications, from cellular differentiation to drug development, due to their ability to more closely replicate the in vivo environment than $2 \mathrm{D}$ models are currently able. ${ }^{97}$ While $2 \mathrm{D}$ cultures exist in a single plane and can be captured by a single image, 3D cultures are distributed over a number of planes in $z$-direction; as such, analysis of 3D cultures has proved difficult as most techniques currently in use were developed for $2 \mathrm{D}$ cultures. To image 3D cultures, a number of images need to be acquired at different focal positions ( $z$-stack), and as confocal Raman spectroscopy is able to do this, it is an attractive option for the 3D imaging of cell cultures. ${ }^{98}$ A good example of this approach is demonstrated by Majzner et al. (Fig. 7) who successfully used confocal Raman spectroscopy for the 3D mapping of individual endothelial cells in vitro and ex vivo within the vascular wall. ${ }^{99}$

SERS nanotags have also been utilised to construct a 3D image of Chinese Hamster Ovarian cells, demonstrating the potential of SERS tags in detecting and mapping intracellular structures. ${ }^{100}$ While in both of these studies, cells were fixed, they show that Raman spectroscopy is a viable technique for 3D imaging in a non-disruptive manner that, if applied to live cells, could lead to an increased understanding of cellular dysfunction in disease by detecting changes in the underlying architecture. In the case of endothelial cells, it could lead to the development of suitable models for vascular diseases, and outlines the potential of 3D Raman mapping in diagnostics. 

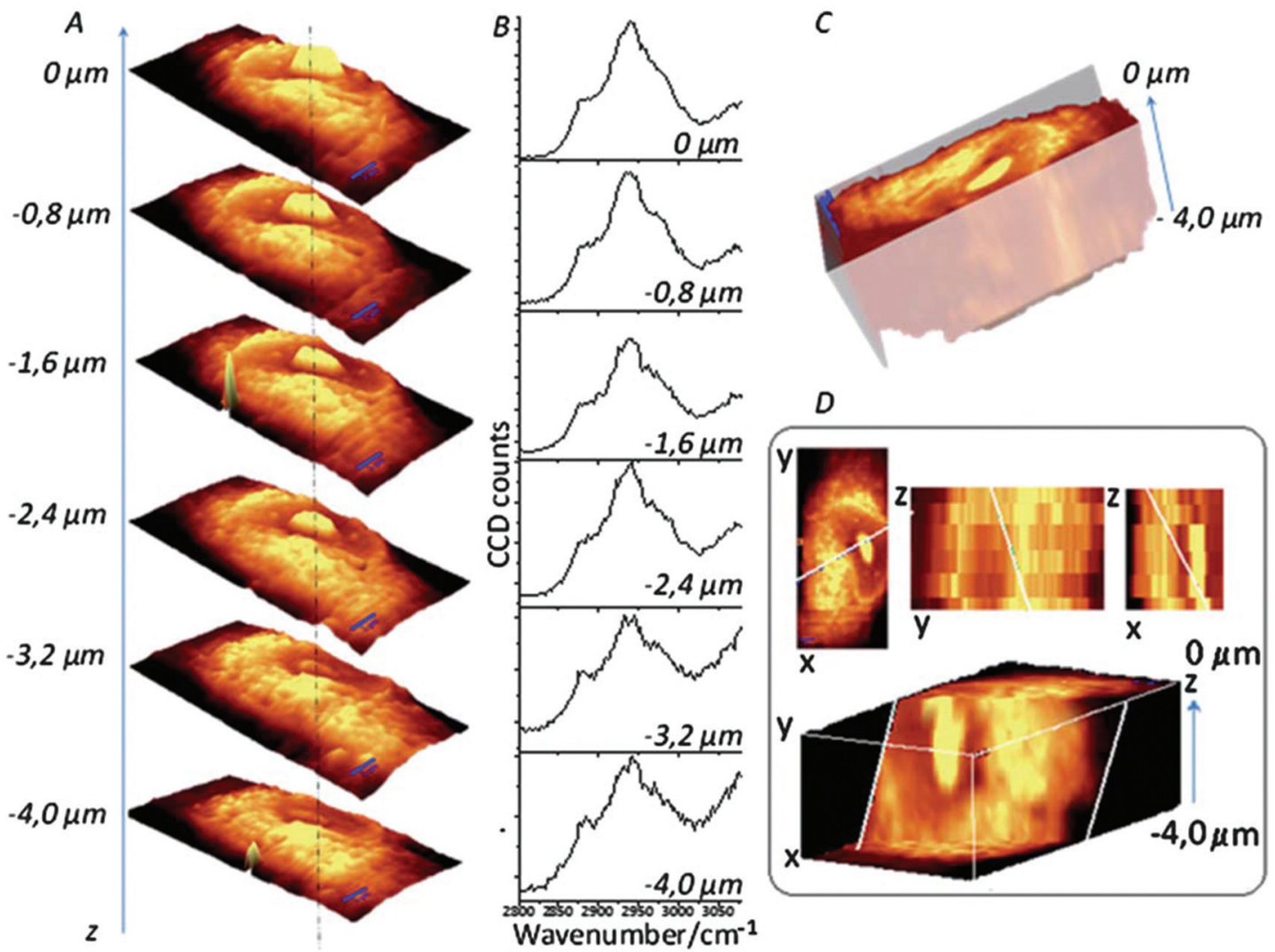

Fig. 7 Confocal Raman imaging-stack of an EA.hy 926 cell covering $16.7 \times 34.1 \times 4 \mu \mathrm{m}^{3}$. Integration map (A) over the $\nu_{\mathrm{C}-\mathrm{H}}$ band $\left(2800-3020 \mathrm{~cm}^{-1}\right.$ range), single spectra (B) extracted from the same point in the maps (dotted line), a reconstructed 3D image seen from the top (C) and a crosssection of the reconstructed 3D image (D). Reproduced with permission from the Royal Society of Chemistry, Copyright 2013, ref. 99.

SERS has been used for the 3D imaging of cellular pathways within living cells. A gold nanoparticle was endocytosed by macrophages and its movement tracked over time to show interactions with intracellular structures associated with cellular transport e.g. microtubule-associated proteins dynein and kinesin. ${ }^{101}$ The ability to track intracellular movement and measure the interaction between nanoparticles and intracellular molecules in 3D is an exciting prospect in cell-based studies, but particularly in drug development as it may allow for the visualisation of drugs of interest in a 3D environment close to in vivo conditions.

\section{Conclusion}

To summarise, Raman spectroscopy is a spectroscopic technique that detects molecular vibrations in order to characterise the underlying chemical structure of a sample. It is particularly relevant in biology as it allows for the non-invasive, labelfree observation and imaging of cells under normal physiological conditions in a way that current imaging techniques cannot. Raman imaging, especially live cell imaging, is a developing technique, and as we have demonstrated already shows potential application in a number of different biomedical fields. These include (but are not limited to): cell characteris- ation and sorting; the development and testing of new pharmaceutical compounds; the diagnosis and detection of disease; and in 3D imaging. While there are still some limitations of Raman spectroscopy, such as the time it takes to acquire maps and a lack of robustness and consistency in the way Raman maps are reported, ${ }^{34}$ there is hope that new developments and advancements can overcome this and lead to Raman spectroscopy becoming a more accessible and widelyused imaging technique in the future.

\section{References}

1 H. Lodish, C. Kaiser, A. Bretscher, A. Amon, A. Berk, M. Krieger, H. Ploegh and M. Scott, Molecular Cell Biology, W. H. Freeman, 7th edn, 2012.

2 C. V. Raman and K. S. Krishnan, Nature, 1928, 121, 501502.

3 E. Smith and G. Dent, Modern Raman Spectroscopy - A Practical Approach, John Wiley \& Sons, Chichester, 2005.

4 A. F. Palonpon, M. Sodeoka and K. Fujita, Curr. Opin. Chem. Biol., 2013, 17, 708-715.

5 A. J. Koster and J. Klumperman, Nat. Rev. Mol. Cell Biol., 2003, Ss6-Ss10, DOI: 10.1038/nrm1194.

6 D. J. Stephens and V. J. Allan, Science, 2003, 300, 82-86. 
7 S. M. Wilson and A. Bacic, Nat. Protoc., 2012, 7, 17161727.

8 R. Henriques, C. Griffiths, E. H. Rego and M. M. Mhlanga, Biopolymers, 2011, 95, 322-331.

9 S. T. Hess, T. J. Gould, M. V. Gudheti, S. A. Maas, K. D. Mills and J. Zimmerberg, Proc. Natl. Acad. Sci. U. S. A., 2007, 104, 17370-17375.

10 R. Wombacher, M. Heidbreder, S. van de Linde, M. P. Sheetz, M. Heilemann, V. W. Cornish and M. Sauer, Nat. Methods, 2010, 7, 717-719.

11 S. A. Jones, S. H. Shim, J. He and X. W. Zhuang, Nat. Methods, 2011, 8, 499-U496.

12 R. B. Sekar and A. Periasamy, J. Cell Biol., 2003, 160, 629633.

13 K. Xu, H. P. Babcock and X. W. Zhuang, Nat. Methods, 2012, 9, 185-188.

14 E. M. Denholm and G. P. Stankus, Cytometry, 1995, 19, 366-369.

15 K. B. Abbitt, G. E. Rainger and G. B. Nash, J. Immunol. Methods, 2000, 239, 109-119.

16 S. M. Ali, F. Bonnier, H. Lambkin, K. Flynn, V. McDonagh, C. Healy, T. C. Lee, F. M. Lyng and H. J. Byrne, Anal. Methods, 2013, 5, 2281-2291.

17 D. H. Rank and R. V. Wiegand, J. Opt. Soc. Am., 1946, 36, 325-334.

18 R. F. Stamm and C. F. Salzman, J. Opt. Soc. Am., 1953, 43, 126-137.

19 M. Minsky, Microscopy Apparatus, US3013467, 1961.

20 A. L. Schawlow and C. H. Townes, US2929922, 1960.

21 P. D. Maker and R. W. Terhune, Phys. Rev. Online Archive, 1965, 137, A801-A818.

22 M. Fleischmann, P. J. Hendra and A. J. McQuillan, Chem. Phys. Lett., 1974, 26, 163-166.

23 M. Delhaye and P. Dhamelincourt, J. Raman Spectrosc., 1975, 3, 33-43.

24 P. C. Lee and D. Meisel, J. Phys. Chem., 1982, 86, 33913395.

25 S. B. Dierker, C. A. Murray, J. D. Legrange and N. E. Schlotter, Chem. Phys. Lett., 1987, 137, 453-457.

26 G. J. Puppels, F. F. de Mul, C. Otto, J. Greve, M. RobertNicoud, D. J. Arndt-Jovin and T. M. Jovin, Nature, 1990, 347, 301-303.

27 S. Nie, Science, 1997, 275, 1102-1106.

28 K. Kneipp, Y. Wang, H. Kneipp, L. T. Perelman, I. Itzkan, R. R. Dasari and M. S. Feld, Phys. Rev. Lett., 1997, 78, 1667-1670.

29 A. Zumbusch, G. R. Holtom and X. S. Xie, Phys. Rev. Lett., 1999, 82, 4142-4145.

30 C. L. Evans, E. O. Potma, M. Puoris'haag, D. Cote, C. P. Lin and X. S. Xie, Proc. Natl. Acad. Sci. U. S. A., 2005, 102, 16807-16812.

31 G. J. Puppels, J. H. F. Olminkhof, G. M. J. Segers-Nolten, C. Otto, F. F. M. De Mul and J. Greve, Exp. Cell Res., 1991, 195, 361-367.

32 A. Zoladek, F. Pascut, P. Patel and I. Notingher, Spectroscopy, 2010, 24, 131-136.
33 S. Schlucker, M. D. Schaeberle, S. W. Huffman and I. W. Levin, Anal. Chem., 2003, 75, 4312-4318.

34 L. Ashton, K. A. Hollywood and R. Goodacre, Analyst, 2015, 140, 1852-1858.

35 W. J. Howat and B. A. Wilson, Methods, 2014, 70, 12-19.

36 N. Uzunbajakava, A. Lenferink, Y. Kraan, E. Volokhina, G. Vrensen, J. Greve and C. Otto, Biophys. J., 2003, 84, 3968-3981.

37 N. Uzunbajakava, A. Lenferink, Y. Kraan, B. Willekens, G. Vrensen, J. Greve and C. Otto, Biopolymers, 2003, 72, 1-9.

38 H. Manen, Y. Kraan, D. Roos and C. Otto, Proc. Natl. Acad. Sci. U. S. A., 2005, 102, 10159-10164.

39 M. Okada, N. I. Smith, A. F. Palonpon, H. Endo, S. Kawata, M. Sodeoka and K. Fujita, Proc. Natl. Acad. Sci. U. S. A., 2012, 109, 28-32.

40 C. Matthäus, S. Boydston-White, M. Miljković, M. Romeo and M. Diem, Appl. Spectrosc., 2006, 60, 1-8.

41 C. Krafft, T. Knetschke, R. H. W. Funk and R. Salzer, Anal. Chem., 2006, 78, 4424-4429.

42 A. S. Haka, K. E. Shafer-Peltier, M. Fitzmaurice, J. Crowe, R. R. Dasari and M. S. Feld, Cancer Res., 2002, 62, 53755380 .

43 J. Chan, D. Taylor and D. Thompson, Biopolymers, 2009, 91, 132-139.

44 A. N. Kuzmin, A. Pliss and P. N. Prasad, Anal. Chem., 2014, 10909-10916.

45 F. Draux, C. Gobinet, J. Sulé-Suso, A. Trussardi, M. Manfait, P. Jeannesson and G. D. Sockalingum, Anal. Bioanal. Chem., 2010, 397, 2727-2737.

46 A. D. Meade, C. Clarke, F. Draux, G. D. Sockalingum, M. Manfait, F. M. Lyng and H. J. Byrne, Anal. Bioanal. Chem., 2010, 396, 1781-1791.

47 B. R. Wood, L. Hammer, L. Davis and D. McNaughton, J. Biomed. Opt., 2005, 10, 14005-14005.

48 I. Notingher, S. Verrier, H. Romanska, A. E. Bishop, J. M. Polak and L. L. Hench, Spectroscopy, 2002, 16, 43-51.

49 F. Draux, P. Jeannesson, A. Beljebbar, A. Tfayli, N. Fourre, M. Manfait, J. Sulé-Suso and G. D. Sockalingum, Analyst, 2009, 134, 542-548.

50 K. Klein, A. M. Gigler, T. Aschenbrenner, R. Monetti, W. Bunk, F. Jamitzky, G. Morfill, R. W. Stark and J. Schlegel, Biophys. J., 2012, 102, 360-368.

51 A. J. Hobro, N. Pavillon, K. Fujita, M. Ozkan, C. Coban and N. I. Smith, Analyst, 2015, 140, 2350-2359.

52 H. J. van Manen, N. Uzunbajakava, R. van Bruggen, D. Roos and C. Otto, J. Am. Chem. Soc., 2003, 125, 12112-12113.

53 H. J. van Manen, Y. M. Kraan, D. Roos and C. Otto, J. Phys. Chem. B, 2004, 108, 18762-18771.

54 S. E. Ong, B. Blagoev, I. Kratchmarova, D. B. Kristensen, H. Steen, A. Pandey and M. Mann, Mol. Cell Proteomics, 2002, 1, 376-386.

55 H. N. N. Venkata and S. Shigeto, Chem. Biol., 2012, 19, 1373-1380.

56 C. Matthaus, C. Krafft, B. Dietzek, B. R. Brehm, S. Lorkowski and J. Popp, Anal. Chem., 2012, 84, 8549-8556. 
57 M. Q. Li, W. E. Huang, C. M. Gibson, P. W. Fowler and A. Jousset, Anal. Chem., 2013, 85, 1642-1649.

58 A. Naemat, H. M. Elsheikha, R. A. Boitor and I. Notingher, Sci. Rep., 2016, 6, DOI: 10.1038/srep20811.

59 F. El-Diasty, Vib. Spectrosc., 2011, 55, 1-37.

60 X. Nan, J.-X. Cheng and X. S. Xie, J. Lipid Res., 2003, 44, 2202-2208.

61 J.-X. Cheng, Y. K. Jia, G. Zheng and X. S. Xie, Biophys. J., 2002, 83, 502-509.

62 P. L. Stiles, J. A. Dieringer, N. C. Shah and R. P. Van Duyne, Annu. Rev. Anal. Chem., 2008, 1, 601-626.

63 A. F. Palonpon, J. Ando, H. Yamakoshi, K. Dodo, M. Sodeoka, S. Kawata and K. Fujita, Nat. Protoc., 2013, 8, 677-692.

64 B. Kang, L. A. Austin and M. A. El-Sayed, Nano Lett., 2012, 12, 5369-5375.

65 J. W. Kang, P. T. C. So, R. R. Dasari and D.-K. Lim, Nano Lett., 2015, 15(3), 1766-1772.

66 K. Kong, C. J. Rowlands, H. Elsheikha and I. Notingher, Analyst, 2012, 137, 4119-4122.

67 L. B. Kong, P. F. Zhang, J. Yu, P. Setlow and Y. Q. Li, Appl. Phys. Lett., 2011, 98, 213703.

68 I. J. Fox, G. Q. Daley, S. A. Goldman, J. Huard, T. J. Kamp and M. Trucco, Science, 2014, 345, 1247391.

69 B. E. Reubinoff, M. F. Pera, C. Y. Fong, A. Trounson and A. Bongso, Nat. Biotechnol., 2000, 18, 559-559.

70 F. C. Pascut, H. T. Goh, N. Welch, L. D. Buttery, C. Denning and I. Notingher, Biophys. J., 2011, 100, 251259.

71 F. C. Pascut, H. T. Goh, V. George, C. Denning and I. Notingher, J. Biomed. Opt., 2011, 16, 045002.

72 A. Ghita, F. C. Pascut, M. Mather, V. Sottile and I. Notingher, Anal. Chem., 2012, 84, 3155-3162.

73 A. Mitchell, L. Ashton, X. B. B. Yang, R. Goodacre, A. Smith and J. Kirkham, Cytometry, Part A, 2015, 87, 1012-1019.

74 A. Mitchell, L. Ashton, X. B. B. Yang, R. Goodacre, M. J. Tomlinson, A. Smith and J. Kirkham, Analyst, 2015, 140, 7347-7354.

75 M. Marti, L. Mulero, C. Pardo, C. Morera, M. Carrio, L. Laricchia-Robbio, C. R. Esteban and J. C. I. Belmonte, Nat. Protoc., 2013, 8, 223-253.

76 A. Zoladek, F. C. Pascut, P. Patel and I. Notingher, J. Raman Spectrosc., 2011, 42, 251-258.

77 K. Bräutigam, T. Bocklitz, M. Schmitt, P. Rösch and J. Popp, ChemPhysChem, 2013, 14, 550-553.

78 H. Salehi, E. Middendorp, I. Panayotov, P.-Y. C. Dutilleul, A.-G. Vegh, S. Ramakrishnan, C. Gergely and F. Cuisinier, J. Biomed. Opt., 2013, 18, 56010-56010.

79 G. Han, R. Liu, M. Y. Han, C. Jiang, J. Wang, S. Du, B. Liu and Z. Zhang, Anal. Chem., 2014, 86, 11503-11507.

80 W. Fang, Z. Wang, S. Zong, H. Chen, D. Zhu, Y. Zhong and Y. Cui, Biosens. Bioelectron., 2014, 57, 10-15.
81 O. Warburg, F. Wind and E. Negelein, J. Gen. Physiol., 1927, 8, 519-530.

82 A. Lattermann, C. Matthäus, N. Bergner, C. Beleites, B. F. Romeike, C. Krafft, B. R. Brehm and J. Popp, J. Biophotonics, 2013, 6, 110-121.

83 K. Marzec, T. Wrobel, A. Rygula, E. Maslak, A. Jasztal, A. Fedorowicz, S. Chlopicki and M. Baranska, Biophotonics, 2014, 7, 744-756.

84 R. Kumar, G. Singh, K. Grønhaug, N. Afseth, C. de Lange Davies, J. Drogset and M. Lilledahl, Int. J. Mol. Sci., 2015, 16, 9341-9353.

85 K. M. Omberg, J. C. Osborn, S. L. L. Zhang, J. P. Freyer, J. R. Mourant and J. R. Schoonover, Appl. Spectrosc., 2002, 56, 813-819.

86 J. W. Chan, D. S. Taylor, T. Zwerdling, S. M. Lane, K. Ihara and T. Huser, Biophys. J., 2006, 90, 648-656.

87 S. Lee, H. Chon, M. Lee, J. Choo, S. Y. Shin, Y. H. Lee, I. J. Rhyu, S. W. Son and C. H. Oh, Biosens. Bioelectron., 2009, 24, 2260-2263.

88 J. Yang, Z. Wang, S. Zong, C. Song, R. Zhang and Y. Cui, Anal. Bioanal. Chem., 2012, 402, 1093-1100.

89 X. Qian, X.-H. Peng, D. O. Ansari, Q. Yin-Goen, G. Z. Chen, D. M. Shin, L. Yang, A. N. Young, M. D. Wang and S. Nie, Nat. Biotechnol., 2008, 26, 83-90.

90 U. S. Dinish, G. Balasundaram, Y.-T. Chang and M. Olivo, Sci. Rep., 2014, 4, 4075-4075.

91 M. S. Bergholt, W. Zheng, K. Lin, K. Y. Ho, M. Teh, K. G. Yeoh, J. B. So and Z. Huang, Technol. Cancer Res. Treat., 2011, 10, 103-112.

92 D. M. Good, V. Thongboonkerd, J. Novak, J.-L. Bascands, J. P. Schanstra, J. J. Coon, A. Dominiczak and H. Mischak, J. Proteome Res., 2007, 6, 4549-4555.

93 C. M. MacLaughlin, N. Mullaithilaga, G. Yang, S. Y. Ip, C. Wang and G. C. Walker, Langmuir, 2013, 29, 1908-1919.

94 S. Li, L. Li, Q. Zeng, Y. Zhang, Z. Guo, Z. Liu, M. Jin, C. Su, L. Lin, J. Xu and S. Liu, Sci. Rep., 2015, 5, 9582-9582.

95 A. Y. Lau, L. P. Lee and J. W. Chan, Lab Chip, 2008, 8, 1116-1120.

96 S. Dochow, C. Beleites, T. Henkel, G. Mayer, J. Albert, J. Clement, C. Krafft and J. Popp, Anal. Bioanal. Chem., 2013, 405, 2743-2746.

97 M. Ravi, V. Paramesh, S. R. Kaviya, E. Anuradha and F. D. P. Solomon, J. Cell. Physiol., 2015, 230, 16-26.

98 V. Charwat, K. Schütze, W. Holnthoner, A. Lavrentieva, R. Gangnus, P. Hofbauer, C. Hoffmann, B. Angres and C. Kasper, J. Biotechnol., 2015, 205, 70-81.

99 K. Majzner, A. Kaczor, N. Kachamakova-Trojanowska, A. Fedorowicz, S. Chlopicki and M. Baranska, Analyst, 2013, 138, 603-610.

100 S. McAughtrie, K. Lau, K. Faulds and D. Graham, Chem. Sci., 2013, 4, 3566-3566.

101 K. C. Huang, K. Bando, J. Ando, N. I. Smith, K. Fujita and S. Kawata, Methods, 2014, 68, 348-353. 